\title{
Energy Efficient MAC-ROUTE Cross-Layer Technique with Competent Scheduling Duty Cycle Protocol for a Multi-Hop WSN
}

\author{
P. M. Prathibhavani and T. G. Basavaraju
}

\begin{abstract}
In Wireless Sensor Network (WSN) sensor nodes are compactly placed and the topology of network changes regularly. Sensor nodes are tiny devices limited in power, computation capacity, memory, sensing range and mobility. Because of wireless and specific application nature of Wireless Sensor Network, traditional layered protocol architecture is found to be less efficient, thus need of cross layer design. This paper explores to optimize the energy consumption across physical, MAC and network layers to prolong the life time of WSN.

Even though Multi-hop Low Energy Adaptive Clustering Hierarchy (MLEACH) is cross layer protocol uses contention free TDMA as a MAC layer, which is difficult to schedule packets if the network is densely deployed and high contention because of more control overhead and loss of data packets. In this paper, we design and implement Energy efficient MAC-ROUTE cross layer technique with competent scheduling duty cycle to address high contention in dense network by reducing control overhead and loss of data packet called Cross-MLEACH. The given mathematical model and simulation results shows that Cross-MLEACH design optimizes energy consumption across MAC and network layers and provides better performance than MLEACH in terms of network life time and significant delay using MATLAB.
\end{abstract}

Index Terms-WSN, MAC-ROUTE, MLEACH, Cross-MLEACH, cross layer.

\section{INTRODUCTION}

In most of WSN, sensor nodes have non-replenishable energy sources and thus energy is a sparse resource in WSN. It is very important that minimizing energy consumption across all layer of protocol to optimize the overall network energy consumption and improve the lifetime of network.

There are many WSN routing protocols among them Low Energy Adaptive Clustering Hierarchy (LEACH) is the primary protocol to reduce energy consumption using clustering hierarchy [1] LEACH protocol will have two phases, setup phase and steady state phase where steady state phase is longer than setup phase. In setup phase cluster heads are elected based on randomly generating value between 0 and 1 . If the generated value is less than threshold value $T(n)$ then node will be elected as cluster head otherwise remains as member node. Sensor node is elected as cluster head $(\mathrm{CH})$ depends on threshold value $T(n)$ as given in formula (1). In

Manuscript received February 20, 2017; revised April 12, 2017.

P. M. Prathibhavani is with the Acharya Institute of Technology, India (e-mail: prathibhavanilamani@gmail.com).

T. G. Basavaraju is with Govt. SKSJTI, India (e-mail: tg.braju@gmail.com). steady state phase cluster head collects data from member nodes, aggregates and transmit data directly to base station.

$$
T(n)=\left\{\begin{array}{l}
\frac{P}{1-P *\left(r \bmod \frac{1}{P}\right)} \text { if } n \in G \\
0 \quad \text { otherwise }
\end{array}\right\}
$$

where: $T(n)=$ Threshold

$\mathrm{P}=$ Desired percentage of $\mathrm{CH}$

$\mathrm{r}=$ Current round

$\mathrm{G}=$ Set of Nodes not $\mathrm{CH}$ in $1 / \mathrm{P}$ rounds.

For LEACH energy consumption as follows: Energy consumption to transmit $\left(E_{C}^{T}\right) k$ bits of data over a distance $d$ is calculated as

$$
\begin{aligned}
& E_{C}^{T}=E_{\text {ele }} \times k+\varepsilon_{\text {amp }} \times k \times d^{2}, d<d_{0} \\
& E_{C}^{T}=E_{\text {ele }} \times k+\varepsilon_{\text {amp }} \times k \times d^{4}, d>d_{0}
\end{aligned}
$$

where: $E_{\text {elec }}=$ Energy consumption to run transmitter node. $\varepsilon_{\text {amp }}=$ Energy consumption for amplification. $k=$ Number of bits in a Message.

Similarly energy consumption to receive $\left(E_{C}^{R}\right)$ and aggregate $\left(E_{C}^{A g g}\right) k$ bits of data is given as

$$
\begin{gathered}
E_{C}^{R}=E_{\text {ele }} \times k \\
E_{C}^{A g g}=E_{\text {Agg }} \times k
\end{gathered}
$$

Then total consumption of energy $E_{C}^{\text {total }}$ is given by

$$
E_{C}^{\text {total }}=E_{C}^{T}+E_{C}^{R}+E_{C}^{A g g}
$$

In LEACH, if cluster head is far from base station energy dissipation is more compare to nearer cluster head. To overcome this, multi-hop routing was designed to minimize energy consumption [2]-[6]. Multi-hop LEACH is the LEACH adaptation routing protocol in which self-elected cluster heads collect, aggregate and transmits data through intermediate CH's to base station using multi-hop. Multi-hop 
LEACH was designed to consume minimum energy when compare to existing LEACH protocol. For Multi-hop LEACH energy consumption as follows:

In Multi-hop LEACH, each cluster contain $p$ number of member nodes and energy consumption of each Member Node and Cluster Head is $E_{C \rightarrow M N}$ and $E_{C \rightarrow C H}$ respectively, then total energy consumption $\left(E_{C_{-} R E L A Y}^{\text {total }}\right)$ of clusters is given as below

$$
\left(E_{C_{-} R E L A Y}^{\text {total }}\right)=E_{C \rightarrow C H}+p \times E_{C \rightarrow M N}
$$

Comparing equation (6) and equation (7) came to conclusion that total network energy consumption is more in single hop LEACH when compare to Multi-hop LEACH [7].

Even though Multi-hop LEACH protocol is cross layer protocol uses contention free TDMA as a MAC layer protocol, which is difficult to schedule packets if the network is densely deployed and high contention because of more control overhead and loss of data packets. In this paper, we design and implement Energy efficient MAC-ROUTE cross layer technique with competent scheduling duty cycle to address high contention in dense network by reducing control overhead and loss of data packet called Cross-MLEACH. The simulation and mathematical results show that Cross-MLEACH protocol resolves the mentioned problem and provides better performance than Multi-hop LEACH in terms of network life time and delay using MATLAB.

The rest of the paper is divided as follows, section II deals with related works. In section III, The proposed work Cross-MLEACH is presented. Section IV is about the mathematical modeling and simulation results using MATLAB and finally section $\mathrm{V}$ is about conclusion.

\section{RELATED WORK}

In Low Energy Adaptive Clustering Hierarchy (LEACH) is the primary Wireless Sensor Network routing protocol to reduce energy consumption using clustering hierarchy. LEACH [2], [8] protocol will have two phases, setup phase and steady state phase where steady state phase is longer than setup phase. In setup phase cluster heads are elected based on randomly generating value between 0 and 1 . If the generated value is less than threshold value $t(n)$ then node will be elected as cluster head otherwise remains as normal node. In steady state phase cluster heads collect data from member nodes, aggregate and transmit data directly to base station. To improve the lifetime of network cross layer technique has to incorporate to LEACH.

MAC-ROUTE cross layer technique is the best to improve lifetime of network and network throughput compare to other cross layer techniques [9]. Here observed that MAC layer plays a vital role in minimizes the overall network energy consumption. Selection of cluster head depends on node position, residual energy and neighbor number. After cluster head selection, optimize data communication using cross layer [10]. Here in order to overcome hotspot problem due to multi-hop, used cross layer, which makes some nodes as relay nodes of communication between other cluster head nodes and base station. This algorithm enhances the lifetime of network.

In order to improve lifetime of network, the network divided into different layer of clusters. Member nodes join cluster heads depends on received signal strength indicator. Once cluster heads selected, collaborates cluster heads with adjacent layers to transmit data to base station using multi-hop called MR LEACH [5]. In order to reduce energy consumption and end to end delay many MAC layer protocols have been proposed in multi-hop WSN's. Sensor MAC protocol (SMAC) was introduced to minimize the energy by turning off radio during idle times. The node sends a single packet over single hop during entire duty cycle hence the latency was increased significantly [11], [12]. In order to overcome SMAC protocol increased latency DC-SMAC (Dynamic Duty Cycle SMAC) was presented. This protocol can predict the network traffic to dynamically adjust the duty cycle can save the energy and reduce the delay [13]. In [14]-[21], Synchronization Window (SW) is used to periodically broadcast SYNC packet to maintain synchronization among nodes. In Data transmission Window (DW), data packets are forwarded to its next hop receiver and forward the same further to schedule multi-hop data packet transmission.

Control frame can transmit across multiple hops to schedule the upcoming data packet along the route. If contention is high due to wireless nature of network, RMAC handle high traffic by moving contention away from busy area and delivering packets in a single cycle [15]. For wireless networks PRMAC protocol enables a node to schedule multi-hop transmission of multiple data packets to be sent to same destination in a cycle. Here in multi-hop scenario degrades both Packet Delivery Ratio and the end to end transmission delay [20].

In addition to find an energy efficient cross layer technique scheduling duty cycle for Multi-hop LEACH, simulation parameters are required to be optimize to get better performance with energy efficient alternative cross layer technique. There is limited literature available on the optimization of energy efficient MAC-ROUTE cross layer MLEACH and hence this research work aims to optimize energy efficient parameters. In this paper we design and implement Energy efficient MAC-ROUTE cross layer technique with competent scheduling duty cycle for Multi-hop LEACH protocol called Cross-MLEACH to address above mentioned problem of delay, control overhead and network life time.

\section{PROPOSED WORK}

Below Fig. 1 shows the idea of our work. Here physical layer obtains the Receiver Signal Strength Indication (RSSI) [22] depends on distance of neighbor node and maintains neighbor table and provides neighbor table information to network layer.

Network layer obtains the neighbor table information from physical layer and construct routing table with final destination address available in respective node and transmits the same to MAC layer. MAC layer with efficient duty cycle schedules the next coming data packet. With the help of this we can handle control overhead and high contention with less packet loss to improve network lifetime. Cross-MLEACH 
protocol works mainly on 2 phases

- Cluster setup phase

- Cluster communication phase

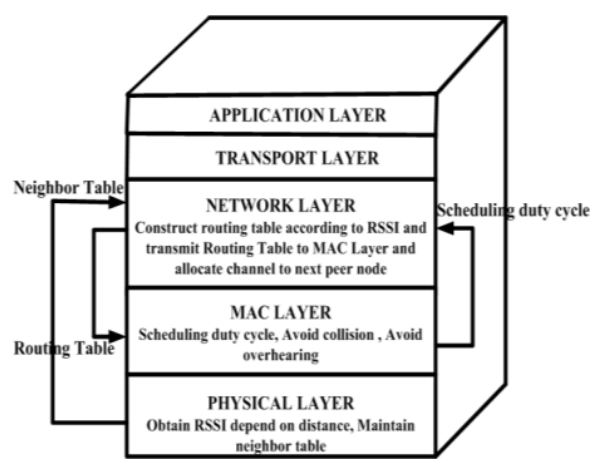

Fig. 1. Idea of proposed work.

\section{A. Cluster Setup Phase}

In Cluster setup phase, each node in network generates a random value between 0 and 1 and compares generated random value with threshold value $T(n)$. If the generated random value is less than $T(n)$ then the node is elected as cluster head $(\mathrm{CH})$ otherwise remain as member node $(\mathrm{MN})$. Each $\mathrm{CH}$ broadcast an advertisement message (ADV Msg) to all neighbor MN's and wait for join message (JOIN Msg) from MN's to form clusters. Each MN receive ADV Msg from neighbor $\mathrm{CH}$ 's and compares which $\mathrm{CH}$ ADV Msg having good signal strength using RSSI and send JOIN request for that $\mathrm{CH}$. Each $\mathrm{CH}$ creates Routing Table (RT) containing current node address, previous node address and next node address and schedule efficient duty cycle TDMA in communication with MAC layer. Cluster setup has done and ready for cluster communication phase. We design Algorithm 1 for cluster setup phase.

Cluster setup phase: $N=$ Set of all sensing nodes in network, $\mathbf{t}(\mathbf{n})=$

$$
\frac{P}{1-P^{*}\left(r \bmod \frac{1}{P}\right)}
$$

For $(\forall N \in G)$ generates random value b/w 0 and 1

Begin

\{

If (generated random value $<\mathrm{t}(\mathrm{n})$ )

\{

Elected as $\mathrm{CH}$.

Broadcast ADV Msg

Receive JOIN Msg

Create RT

Schedule TDMA

Broadcast TDMA to MN's

\}

Else

\{

Wait for ADV Msg.

Wait TDMA

Send JOIN Msg ( RSSI)

Receive TDMA
End

\}

End

Algorithm 1. Cluster setup phase

\section{B. Cluster Communication Phase}

$>$ Intra cluster communication phase

$>$ Inter cluster communication phase

$\checkmark$ Data scheduling phase

$\checkmark$ Data transmission phase

\section{1) Intra cluster communication phase}

Once cluster setup has been done, intra cluster communication phase starts. Each member node of cluster sense the data and send to respective cluster head. Cluster head receives the data from member nodes according to TDMA slot, $\mathrm{CH}$ aggregates the data and ready for inter cluster communication phase. Algorithm 2 proposed for intra cluster communication phase.

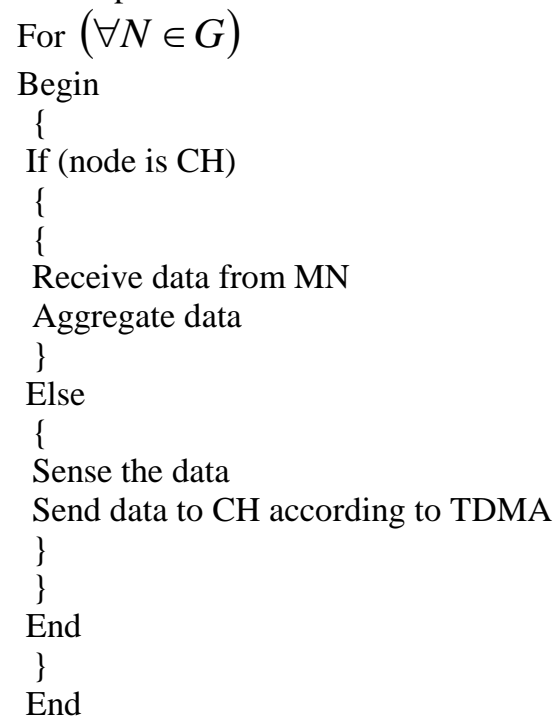

Algorithm 2. Intra cluster communication phase

\section{2) Inter cluster communication phase}

Inter cluster communication phase divided into data scheduling and data transmission phase. In data scheduling phase, each $\mathrm{CH}$ obtains destination (DST) address information from network (NET) layer and creates Routing table and pass it to MAC layer. MAC layer with efficient duty cycle TDMA, schedule the packet along with NET layer information by creating Multi-Hop Control Packet (MHCP) (cur_node_add, prev_node_add, nex_node_add, dst_add). Transmission of MHCP to immediate $\mathrm{CH}$ to inform when to be awake to receive data packets from previous node and transmits to next neighbor $\mathrm{CH}$ node. Data scheduling will be done from each $\mathrm{CH}$ to destination through intermediate $\mathrm{CH}$ 's as shown in Fig. 2 for MLEACH and in Fig. 3 for proposed Cross-MLEACH. Once after data scheduling has done from each $\mathrm{CH}$ to destination, data transmission starts. Algorithm 3 designed for data scheduling phase.

\section{Data scheduling phase:}

$$
\begin{aligned}
& \text { For }(\forall C H \in G) \\
& \text { Begin } \\
& \{
\end{aligned}
$$


Obtains DST Info from NET layer and creates Routing Table

Creates MHCP

Send MHCP to immediate node

Previous step repeats till reaches DST

\} End

Algorithm 3. Data scheduling phase

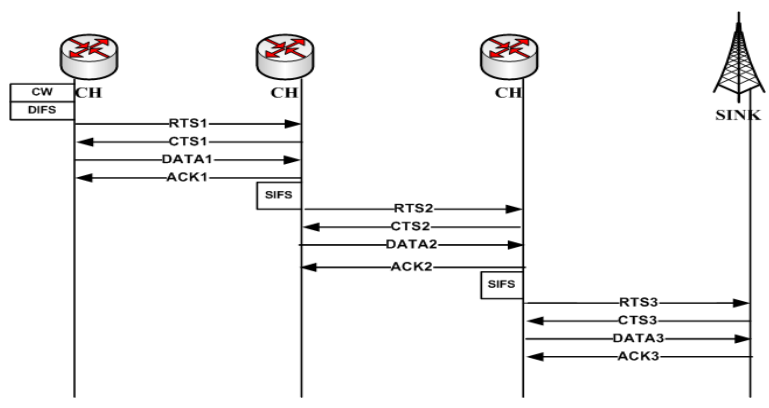

Fig. 2. Data scheduling in MLEACH.

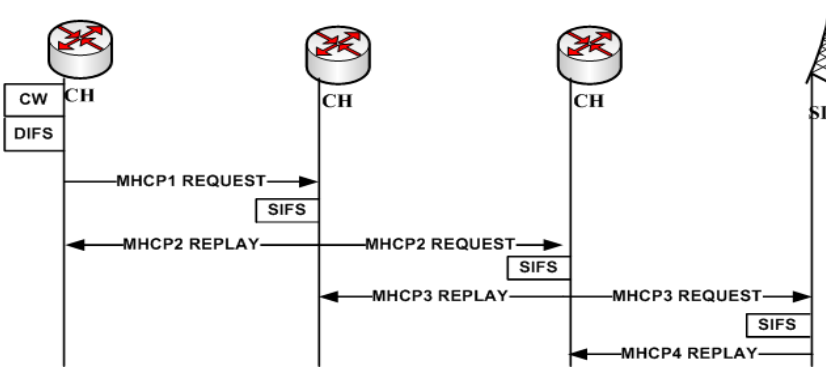

Fig. 3. Data scheduling in proposed Cross-MLEACH.

Each $\mathrm{CH}$ obtains the data scheduling information from MHCP and forwards the data till reaches destination in one operation cycle as shown in Fig. 4. Algorithm 4 for data transmission phase is shown below.

Data transmission phase:

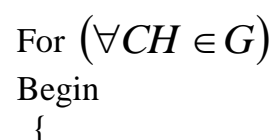

Obtains data scheduling info from MHCP

Forward data till reaches DST in single cycle. \}

End

Algorithm 4. Data transmission phase

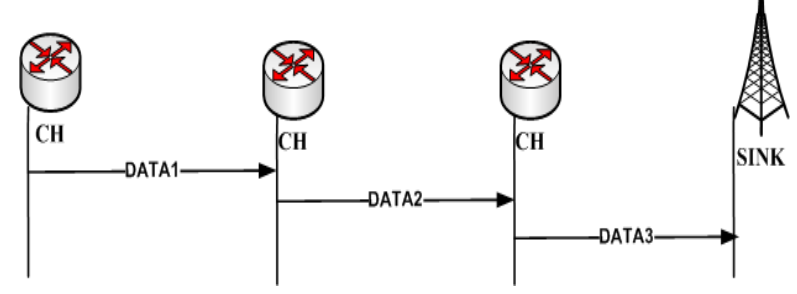

Fig. 4. Data transmission in one operation cycle.

\section{MATHEMATICAL MOdELING AND SimUlation PARAMETERS}

Simulations were done with MATLAB to evaluate
Cross-MLEACH and to compare result with MLEACH to show that Cross-MLEACH can improve lifetime of network. Table I shows basic parameter settings for simulations.

\begin{tabular}{|c|c|}
\hline Parameter & Value \\
\hline Network Size & $200 \mathrm{~m} \times 200 \mathrm{~m}$ \\
\hline Nodes & 150 \\
\hline Base Station Location & $(50,175)$ \\
\hline Cluster Head Percentage(P) & 0.1 \\
\hline Initial energy $\left(\mathrm{E}_{\mathrm{o}}\right)$ & 1 Joule \\
\hline Data Packet Size & 50 bytes \\
\hline Bandwidth & $20 \mathrm{kbps}$ \\
\hline Free Space Loss $\left(\mathrm{E}_{\mathrm{fs}}\right)$ & 10pJoule $/ \mathrm{bit} / \mathrm{m}^{2}$ \\
\hline Multipath Loss $\left(E_{m p}\right)$ & $0.0013 \mathrm{pJoule} / \mathrm{bit} / \mathrm{m}^{4}$ \\
\hline Data Aggregation Energy $\left(\mathrm{E}_{\mathrm{da}}\right)$ & 5pJoule/bit/signal \\
\hline Threshold Distance $\left(\mathrm{d}_{\mathrm{o}}\right)$ & $\operatorname{Sqrt}\left(\mathrm{E}_{\mathrm{fs}} / \mathrm{E}_{\mathrm{mp}}\right)$ \\
\hline Tx/Rx Power & $1 \mathrm{~W}$ \\
\hline Contention Window & $64 \mathrm{~ms}$ \\
\hline Sleep Power & $0.05 \mathrm{~W}$ \\
\hline Idle Power & $0.45 \mathrm{~W}$ \\
\hline DIFS & $10 \mathrm{~ms}$ \\
\hline SIFS & $5 \mathrm{~ms}$ \\
\hline RTS/CTS Frame size & 10bytes \\
\hline ACK Frame size & 10bytes \\
\hline $\begin{array}{l}\text { MHCP(Multi Hop Control Packet) } \\
\text { Frame size }\end{array}$ & 14bytes \\
\hline
\end{tabular}

In our simulation, we are using each data packet of size 50 bytes. The transmission duration for RTS, CTS, ACK and MHCP are calculated as below by using preamble size of default 5 bytes and encoding ratio 2 .

$$
\begin{aligned}
& \text { DurRTS }=\left(\frac{\text { Preamblesize }+\left(T R S_{\text {Framesize }} \times \text { Encoding }_{\text {Ratio }}\right.}{\text { Bandwidth }}\right)+1 m s \\
& \text { DurCTS }=\left(\frac{\text { Preamblesize }+\left(\text { CTS }_{\text {Framesize }} \times \text { Encoding }_{\text {Ratio }}\right.}{\text { Bandwidth }}\right)+1 m s
\end{aligned}
$$

DurACK $=\left(\frac{\text { Preamblesize }+\left(A C K_{\text {Framesize }} \times \text { Encoding }_{\text {Ratio }}\right.}{\text { Bandwidth }}\right)+1 m s$

DurMHCP $=\left(\frac{\text { Preamblesize }+\left(\text { MHCP }_{\text {Framesize }} \times \text { Encoding }_{\text {Ratio }}\right.}{\text { Bandwidth }}\right)+1 m s$

DurDATA $=\left(\frac{\operatorname{Pr} \text { eamblesize }+\left(\text { DATA }_{\text {Framesize }} \times \text { Encoding }_{\text {Ratio }}\right.}{\text { Bandwidth }}\right)+1 m s$

The duration of MLEACH protocol for one complete end to end transmission is calculated as below for $\mathrm{N}$ number of hops:

$T_{\text {Data_Duration }}(\mathrm{MLEACH})=\mathrm{CW}+\mathrm{DIFS}+(\mathrm{N}-1) \mathrm{SIFS}+\mathrm{N} \times($ Dur RTS+DurCTS +DurACK+DurDATA) 
The energy required to transmit $T_{\text {Data_Duration }}$ Data packet of MLEACH protocol for one complete end to end transmission is calculated as below for $\mathrm{N}$ number of hops with $T_{x} / R_{X}$ power of $1 \mathrm{~W}$.

$E_{\text {Data }}(\mathrm{MLEACH})=T_{\text {Data_Duration }}(\mathrm{MLEACH})\left(\mathrm{T}_{\mathrm{X}} / \mathrm{R}_{\mathrm{X} \_ \text {power }}\right)$

The duration of Cross-MLEACH protocol for one complete end to end transmission is calculated as below for $\mathrm{N}$ number of hops:

$T_{\text {Data_duration }}($ Cross-MLEACH $)=\mathrm{CW}+\mathrm{DIFS}+\mathrm{N} \times(2 \times \mathrm{DurMH}$ $\mathrm{CP}+\mathrm{SIFS}+$ DurDATA)

The energy required to transmit $T_{\text {Data_Duration }}$ Data packet of Cross-MLEACH protocol for one complete end to end transmission is calculated as below for $\mathrm{N}$ number of hops with $T_{x} / R_{X}$ power $1 \mathrm{~W}$

$E_{\text {Data }}($ Cross-MLEACH $)=T_{\text {Data_Duration }}($ Cross-MLEACH $)$ $\times\left(T_{X} / R_{X \_ \text {power }}\right)$

Further equations (14) and (16) are compared by substituting simulation parameter shown in table 1 for $\mathrm{N}$ number of hops. Then, energy dissipation is given by

$$
E_{\text {Data }}(\mathrm{MLEACH})>E_{\text {Data }}(\text { Cross-MLEACH })
$$

From Equation (17) it is observed that the energy dissipation in MLEACH is more compared to Cross-MLEACH. Therefore the lifetime of Cross-MLEACH is more and end to end transmission delay is less when compared to MLEACH.

\section{A. Life Time of the Network}

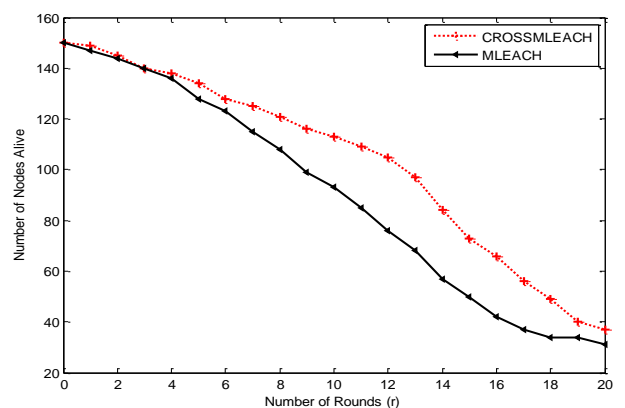

Fig. 5. Comparison number of nodes alive vs. number of rounds.

Fig. 5 shows the total number of alive nodes per the number of rounds. Nodes remain alive long for Cross-MLEACH protocol because Cluster head creates MHCP with final destination address available in network layer of respective node. MHCP will have current node address, previous node address and next node address and schedule the data transmission till reaches sink node. Data transmission starts as data schedule done by MHCP. This helps in schedule the high contention in densely deployed network by reducing control overhead with less packet loss and also maximize the network lifetime compare to MLEACH.

\section{B. Number of Nodes Alive Per Number of Packets}

Received at Base Station (BS)

Fig. 6 shows number of nodes that remain alive per number of packets received at the $\mathrm{BS}$. The number of packets received at BS in MLEACH is 270 packets where in Cross-MLEACH 320 packets because number of alive node per round is more in Cross- MLEACH.

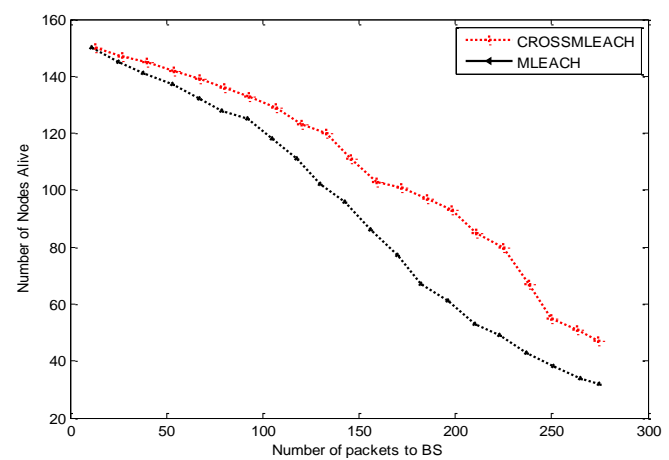

Fig. 6. Number of nodes alive vs. Number of packets received at base station.

\section{Number of Packets Received at Cluster Head (CH) Per Number of Rounds}

Fig. 7 shows number of packets received at the $\mathrm{CH}$ over number of rounds. The number of packets received at $\mathrm{CH}$ in Cross-MLEACH is almost same as MLEACH because of data scheduling with competent duty cycle done only at inter cluster communication phase not at intra cluster communication phase.

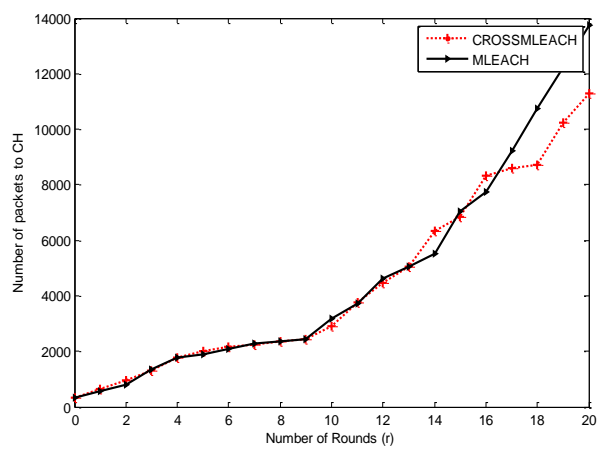

Fig. 7. Number of packets received at cluster head $(\mathrm{CH})$ vs. Number of rounds.

D. Number of Packets Received at Base Station (BS) per Number of Rounds

Fig. 8 shows number of packets received at the BS per number of rounds. The number of packets received at BS is more in Cross-MLEACH compare to MLEACH because number of alive node per round is more in Cross- MLEACH.

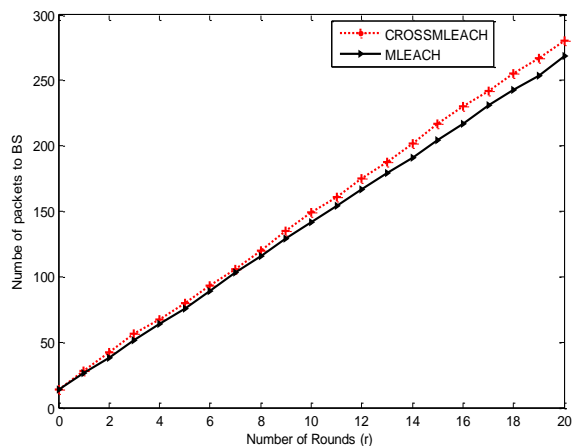

Fig. 8. Number of packets received at base station (BS) vs. Number of rounds. 


\section{CONCLUSION}

In this paper (investigation), mathematical model for energy dissipation of MLEACH and cross-MLEACH is derived and compared. A new MAC-ROUTE cross layer technique is designed with competent duty scheduling mechanism for existing MLEACH protocol to optimize latency and lifetime of wireless sensor network. The simulation and mathematical results show that our proposed protocol Cross-MLEACH optimizes energy consumption across MAC and network layers to provide better performance than MLEACH in terms of network life time and significant end to end delivery using MATLAB.

- Nodes remains maximize network lifetime for Cross-MLEACH protocol.

- Alive node per round is more in Cross- MLEACH and the number of packets received at base station is 320 whereas 270 packets are received for MLEACH protocol.

- Scheduling of the high contention with less delay for Cross-MLEACH protocol compared to MLEACH with minimum control overhead.

- The number of packets received at $\mathrm{CH}$ in Cross-MLEACH is approximately same compared to MLEACH.

- The number of packets received at $\mathrm{BS}$ is more in Cross-MLEACH compared to MLEACH.

\section{REFERENCES}

[1] W. B. Heinzelman, "Application-specific protocol architectures for wireless networks," Ph.D. dissertation, Department of Electrical Engineering and Computer Science, MIT, Cambridge, MA, 2000.

[2] W. R. Heinzelman, A. Chandrakasan, and H. Balakrishnan, "Energy efficient communication protocol for wireless micro-sensor networks,' in Proc. 33rd IEEE, HICSS, 2000.

[3] S. Tanessakulwattana, C. Pornavalai, and G. Chakraborty, "Adaptive multi-hop routing for wireless sensor network," IEEE, JCSSE, pp. 105-110, 2013.

[4] M. Omsar and W. Fateh, "Enhancing multihop routing protocols in wireless sensor networks using LEACH-1R," IEEE, WSWAN, pp. 1-6, 2015.

[5] M. Omar, A. B. Dogar, and G. A. Shah, "Mrleach: Multihop routing with low energy adaptive clustering hierarchy," IEEE, SENSOR COMM, pp. 262-268, 2010.

[6] Z. Jun, Q. Xia, and F. Li, "Research of cross-layer and multi-hops algorithm based on energy and location," IEEE, ICICEE, pp. 1781-1784, 2012.

[7] V. Sharma and D. Saini, "Performance investigation of advanced multi-hop and single-hop energy efficient LEACH protocol with heterogeneous nodes in wireless sensor networks," in Proc. IEEE, ICACCE, 2015, pp. 192-197.

[8] H. Kong, "Energy efficient cooperative leach protocol for wireless sensor networks," IEEE, JCN, pp. 358-365, 2010.

[9] P. M. Prathibhavani and T. G. Basavaraju," Energy efficient cross layer design protocols using distributed method of information sharing in wireless sensor networks- A review," ISAET, IJCSEE, pp. 181-189, 2015.

[10] S. H. Gajjar, K. S. Dasgupta, S. N. Pradhan, and K. M. Vala, "Lifetime improvement of LEACH protocol for wireless sensor network," NUiCONE, pp. 1-6, 2012.

[11] W. Ye, J. Heidemann, and D. Estrin, "An energy-efficient MAC protocol for wireless sensor networks," INFOCOM, pp. 1567-1576, 2002.

[12] W. M. Song, Y. M. Liu, and S. E. Zhang, "Research on SMAC protocol for WSN wireless communications," in Proc. 4th Intl. Conf. on Networking and Mobile Computing (WiCOM), 2008, pp. 1-4.

[13] C. Wang, Y. Chen, and Y. Hou, "The analysis and improvement of SMAC protocol for wireless sensor networks," International
Conference on Mobile Ad-hoc and Sensor Networks (MSN), IEEE, 2013.

[14] W. Ye, J. Heidemann, and D. Estrin, "Medium access control with coordinated adaptive sleeping for wireless sensor networks," IEEE/ACM Trans. on Netw, vol. 12, no. 3, pp. 493-506, 2004.

[15] S. Du, A. Saha, and D. Johnson, "RMAC: A routing enhanced duty cycle MAC protocol for wireless sensor networks," in Proc. INFOCOM, 2007, pp. 1478-1486.

[16] Y. Sun, S. Du, O. Gurewitz, and D. B. Johnson, "DW-MAC: A low latency energy efficient demand-wakeup MAC protocol for wireless sensor networks," in Proc. MobiHoc, 2008, pp. 53-62.

[17] G. Liu and G. Yao, "SRMAC: Staggered routing-enhanced MAC protocol for wireless sensor networks," in Proc. WiCOM, 2011, pp. 1-6.

[18] K. T. Cho and S. Bahk, "HE-MAC: Hop extended MAC protocol for wireless sensor networks," in Proc. GLOBCOM, 2009, pp. 1-6.

[19] K. T. Cho and S. Bahk, "Optimal hop extended MAC protocol for wireless sensor networks," Comput. Netw., vol. 56, no. 4, pp. 1458-1469, 2012.

[20] T. Canli and A. Khokhar, "PRMAC: Pipelined routing enhanced MAC protocol for wireless sensor networks," in Proc. ICC, 2009, pp. 1-5.

[21] R. Singh and S. Chouhan, "A cross-layer MAC protocol for contention reduction and pipelined flow optimization in wireless sensor networks," in Proc. RETIS, 2015, pp. 58-63.

[22] S. Hong and C. Lin, "A cluster routing algorithm based on RSSI for an efficient multi-hop data forwarding," IEEE, ISOCC, pp. 339-340, 2015 .

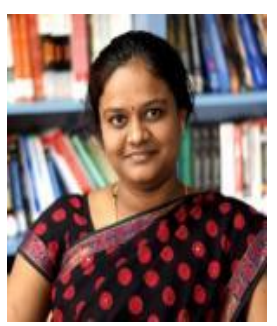

P. M. Prathibhavani is working as an assistant professor in the Department of ISE, Acharya Institute of Technology, Bangalore. She obtained her post-graduation in the Department of Information Technology from UVCE, Bangalore University, Bangalore. Presently, she is pursuing Ph.D under the guidance of Dr. T G Basavaraju in WSN on cross layer. She has more than 8 years of teaching and research experience. She has taught several courses for both UG and PG levels in the area of WSN, electronic circuits, adhoc networks, mobile computing, advanced digital communication, mobile device forensics, cyber space and probability stochastic and queuing theory etc. Her research area is wireless sensor network. She has guided several projects on wireless sensor network for both UG and PG students. She has published more than 20 technical national, international conferences and journals in the area of wireless sensor network. She is a life member of IAENG, ISTE. P. M. Prathibhavani associated with the Old Dominion University, USA as research project coordinator of Department of ISE, Acharya Institute of Technology, Bangalore. She has guided several students in ODU program and sent several students to ODU, USA to get benefit of internship.

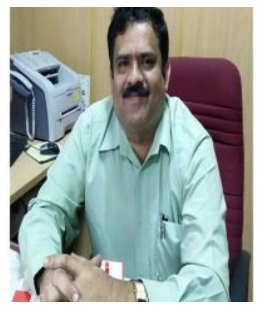

T. G. Basavaraju is currently working as professor and head of CSE Department at Government SKSJ Technological Institute, Bangalore. He is a board of studies member in computer science and engineering stream, VTU, belgaum from the year 2013 to 2017. Basavaraju holds a Ph.D. (Engg.) from Jadavpur University, Kolkata in the area of mobile adhoc networks. He obtained his ME in CSE from UVCE, Bangalore University, Bangalore and secured first rank. He has more than 19 years of experience in teaching and industry. He has authored and co-authored five text books in the area of computer networking. One of his co-authored text book on "mobile wireless ad hoc networks: principles, protocols and applications" was published from auerbach publishers (Taylor and Francis group), USA. He has to his credit more than 55 research publications in international journals and conferences. He is recipient of ISTE National Award instituted by U.P. Government for outstanding work done in specific areas in engineering and technology for the year 2008. He is a life member of ISTE, ACS, CSI, I.E, IAENG. He has served as expert member, NBA of AICTE New Delhi. Also he is a expert member in LIC of VTU, Belgaum and Department of Technical Education, Government of Karnataka. He is a reviewer for IEEE conferences and IEE communication networks journals. 